\title{
Galactic structure studies with BATC star counts
}

\author{
Cuihua $\mathrm{Du}^{1}$, Xu Zhou ${ }^{1}$, Jun $\mathrm{Ma}^{1}$, Alfred Bing-Chih Chen ${ }^{2}$, Yanbin Yang ${ }^{1}, \mathrm{Jiuli} \mathrm{Li}^{1}$, Hong Wu${ }^{1}$, \\ Zhaoji Jiang ${ }^{1}$, and Jiansheng Chen ${ }^{1}$ \\ 1 National Astronomical Observatories, Chinese Academy of Sciences, Beijing 100012, PR China \\ e-mail: dch@vega.bac.pku.edu.cn \\ 2 Department of Physics, National Cheng Kung University, Taiwan 70148, Taiwan
}

Received 16 January 2003/ Accepted 21 March 2003

\begin{abstract}
We report the first results of star counts carried out with the National Astronomical Observatories (NAOC) 60/90 cm Schmidt Telescope in 15 intermediate-band filters from 3000 to $10000 \AA$ in the BATC survey. We analyze a sample of over 1400 main sequence stars $(14 \leq V \leq 21)$, which lie in the field with central coordinates $\mathrm{RA}=09^{\mathrm{h}} 53^{\mathrm{m}} 13.30$ and Dec $=$ $47^{\circ} 49^{\prime} 00^{\prime} 0(\mathrm{~J} 2000)\left(\right.$ Galactic coordinates: $l=169.95^{\circ}, b=49.80^{\circ}$ ). The field of view is $0.95 \mathrm{deg}^{2}$, and the spatial scale was $1^{\prime \prime} 67$. Since star counts at high galactic latitudes are not strongly related to the radial distribution, they are well suited to study the vertical distribution of the Galaxy. In our model, the distribution of stars perpendicular to the plane of the Galaxy is given by two exponential disks (thin disk plus thick disk) and a de Vaucouleurs halo. Also, based on star counts, we derive the scale heights of the thin disk to be $320_{-15}^{+14} \mathrm{pc}$ and of the thick disk to be $640_{-32}^{+30} \mathrm{pc}$, respectively, with a local density of $7.0 \pm 1 \%$ of the thin disk. The errors of scale heights and the corresponding space number density normalization are estimated at a $68 \%$ confidential level. The density law for the Galactic halo population is also investigated. We find that the observed counts support an axial ratio of $c / a \leq 0.6$ for a de Vaucouleurs $r^{1 / 4}$ law, implying a more flattened halo. We consider that it is possible that the halo has two subpopulations-a flattened inner halo and a spherical outer halo in the Milky Way, and such a halo model might resolve many of the divergences in star count results. We also derive the stellar luminosity function (SLF) for the thin disk, and it partly agrees with the Hipparcos luminosity function.
\end{abstract}

Key words. Galaxy: structure - stars: luminosity function, mass function

\section{Introduction}

Over the past decade considerable efforts have been undertaken to gain information about the structure of the Galaxy. For example, Méndez (1996) has developed a Galactic structure model to predict star-counts self-consistently with kinematic distribution of stars; novel multivariate data analysis methods are being applied to recent surveys to characterize the individual stellar distributions of each populations (Chen 1997, 1999); age-metallicity or the kinematics of nearby halo and disk stars have been determined (Norris 1998), and so on. The starcount method which is predominantly used to study the general properties of the Galaxy is a very effective way of constraining global structural parameters for the Milky Way, and of providing density distribution of the Galaxy components.

Bahcall \& Soneira (1980) established the first selfconsistent model by analyzing star count data and derived that the old thin disk has a scale height of $325 \mathrm{pc}$, and that the halo can be represented by a deprojected de Vaucouleurs $r^{1 / 4}$ law. Bahcall \& Soneira $(1984,1985)$ showed that their two major components (thin-disk and spheroid) model for the distribution of stars in the Galaxy is in agreement with all the available data.

Send offprint requests to: Cuihua $\mathrm{Du}$, e-mail: dch@vega.bac.pku.edu.cn
However, Gilmore (1984) pointed out that the star count data cannot be fitted much better by a two component model, and proposed a Galaxy model with three populations: the halo, thin disk and thick disk. At the same time, the scale height of the thick disk was derived by Gilmore (1984) to be 1450 pc with a space number density $2 \%$ of the thin disk in the solar neighborhood. Ojha et al. (1996) found that the vertical distribution of stars fainter than $M_{\mathrm{v}}=3.5$ has scale height $h_{z}=260 \pm 50 \mathrm{pc}$ in the thin disk, and $h_{z}=760 \pm 50 \mathrm{pc}$ in the thick disk with a local density of $7.4 \%$ relative to the thin disk. Chen (2001) used high latitude star counts to derive the scale height of the old thin disk to be $330 \pm 3 \mathrm{pc}$, and the thick disk between $580 \mathrm{pc}$ and 750 pc with a local density of $13 \%-6.5 \%$ of the thin disk in their best-fit model.

For a spherical halo, the standard references for star counts are Kron (1980), Koo \& Kron (1982, 1986) for two fields: SA 57 (near the North Galactic pole) and SA 68 (near $l=$ $90^{\circ}, b=-46^{\circ}$ ). Using a de Vaucouleurs $r^{1 / 4}$ law spheroid, Bahcall \& Soneira (1984) found that this axial ratio was at least 0.8. However, Wyse \& Gilmore (1989) argued on the basis of star counts that the axial ratio for the stellar halo is much flatter than this canonical value. They made an independent analysis of two fields, the south Galactic Pole and one at 
$l=272^{\circ}, b=-44^{\circ}$, and obtained a very different ratio of 0.6 , implying a more flattened halo. Based on star counts from the APS catalog of the POSS, Larsen (1994) gave a mean ratio of 0.5 implying $c / a \leq 0.5$ for the stellar halo.

Although the existence of both the disk and halo components seems well established, the spatial distribution of the Galactic components are not well determined and remain controversial due to different and conflicting results from modeling of star counts. This is unfortunate because the shape of Galactic components are necessary for understanding the conditions during the formation of the Galaxy. In addition, the stellar luminosity function of main sequence stars in the disk is also a topic of debate. For example, there are some arguments about whether features like the Wielen dip near $M_{\mathrm{v}} \sim 7$ mag and the apparent rise of the stellar luminosity function beyond $M_{\mathrm{v}} \sim 12$ exist in the local neighborhood. Therefore, it is worthwhile to know if the features are present in the BATC starcount.

To pin down the Galactic structure with more precision, the Beijing-Arizona-Taiwan-Connecticut (BATC) multicolor photometric survey provides new catalogs with accurate object classification. These catalogs are very useful in constraining the structure of the main components of the Galaxy. In this paper, based on the BATC observation, we investigate the vertical distribution of stars in the Milky way. Details of observations and data reduction are given in Sect. 2; Sect. 3 describes the object classification and the photometric parallaxes; Sect. 4 deals with the space density distribution of stars; Sect. 5 gives the SLF of the thin disk. Finally, we summarize our main conclusions and briefly mention the prospects for future work on this survey project.

\section{BATC Observations and data reduction}

\subsection{BATC photometric system and observations}

The BATC survey performs photometric observations with a large field multicolor system. There are 15 intermediate-band filters in the BATC filter system, which covers an optical wavelength range from 3000 to $10000 \AA$ (Fan et al. 1996; Yan et al. 2000; Zhou 2001). The observation is carried out with the $60 / 90 \mathrm{~cm} \mathrm{f} / 3$ Schmidt Telescope of National Astronomical Observatories (NAOC), located at the Xinglong station with an altitude of $900 \mathrm{~m}$. A Ford Aerospace $2048 \times 2048$ CCD camera with a $15 \mu \mathrm{m}$ pixel size is mounted at the main focus of the Schmidt telescope. The field of view of the CCD is $58^{\prime} \times 58^{\prime}$ with a pixel scale of $11^{\prime \prime} 67$.

As in the definition of the AB system of Oke \& Gunn (1983), the BATC magnitude system is defined as follows:

$m_{\text {batc }}=-2.5 \log \widetilde{F_{v}}-48.60$,

where $\widetilde{F_{v}}$ is the flux per unit frequency in unit of erg s${ }^{-1} \mathrm{~cm}^{-2} \mathrm{~Hz}^{-1}$. The advantage of the $A B$ magnitude system is that the magnitude is directly related to physical units. The 4 Oke \& Gunn (1983) standards are used for flux calibration in the BATC survey. The 4 stars are HD19445, HD84937, BD+262606 and BD+174708. The magnitudes of these standards were refined by several authors. Fukugita et al. (1996) presented the latest re-calibrated fluxes. Their magnitudes were also corrected in the BATC photometric system (Zhou et al. 2001). The extinction coefficients and magnitude zero points obtained from standard star observations are then used to calibrate other BATC field images. The observation for calibration are described in detail in Zhou et al. (2001).

The observations of the BATC T329 field (Galactic coordinates: $\left.l=169.95^{\circ}, b=49.80^{\circ}\right)$ were obtained in 15 intermediate band filters with a total exposure time of 69.37 hours from December 11, 1996 to May 5, 1999. The CCD images are centered at RA $=09^{\mathrm{h}} 53^{\mathrm{m}} 13.30$ and $\operatorname{Dec}=47^{\circ} 49^{\prime} 00^{\prime \prime}(\mathrm{J} 2000)$. The resulting images with the total exposure time and image numbers of each filter band are listed in Table 1.

\subsection{Data reduction}

The BATC survey images were reduced through standard procedures, including bias subtraction, flat-fielding correction and flux calibrations (Fan et al. 1996; Zhou et al. 2001, 2003). After the basic correction described above, the flat-fielded images and field images observed of each filter were combined by integer pixel shifting, respectively. When combining the images, the cosmic rays and bad pixels were corrected by comparison of multiple images. The HST Guide star catalog (GSC) (Jenkner et al. 1990) was then used for coordinate determination. The final RMS error in positions of GSC stars is about 0.5 arcsec.

The magnitude of the point sources in the BATC fields are measured by the photometric method of point spread function (PSF) fitting. Our PSF magnitudes were obtained through an automatic data reduction program PIPELINE 2, which was developed based on Stetson's DAOPHOT procedures (Stetson 1987).

It is difficult to obtain good PSF fitting for faint stars due to their asymmetry in profile. However, in order to construct a SED catalog as complete as possible, faint stars were measured with aperture photometry. The difference between the PSF and aperture photometry result is corrected by the bright, isolated stars in the same field. At the completion of photometry, the SEDs of all measurable objects are obtained. Finally, we determine the SEDs of 15 filters for more than 6000 objects in the BATC T329 field. In Fig. 1, we plot the BATC $d, i, m$ magnitude histograms for the 6000 objects in the field. In general, the limiting magnitude of our photometry is about 21 . 0 with an error of about 0.1 in the BATC $i$ band.

\section{Object classification and photometric parallaxes}

One of the goals of the BATC survey is the classification of every object found in all BATC fields, and the majority of these objects should be classified according to their SED information constructed from the 15-color photometric catalog. The observed colors of each object are compared with a color library of known objects with the same photometric system. For each object the probability of belonging to a certain object class (stars-galaxies) is given. Following that, we use spectral templates of galaxies and stars to discriminate the stars from galaxies. The profile of objects classified as stars does not deviate significantly from that of stellar templates. The input spectral 
Table 1. Parameters of the BATC filters and statistics of observations for the BATC T329 sample (from 1996 to 1999).

\begin{tabular}{cccccccc}
\hline \hline No. & Filter & $\begin{array}{c}\text { Wavelength } \\
(\AA)\end{array}$ & $\begin{array}{c}F W H M \\
(\AA)\end{array}$ & $\begin{array}{c}\text { Total exp. } \\
\text { (hour) }\end{array}$ & $\begin{array}{c}\text { Number of } \\
\text { images }\end{array}$ & $\begin{array}{c}\text { Number of } \\
\text { calibrations }\end{array}$ & $\begin{array}{c}\text { Calib. } \\
\text { error }\end{array}$ \\
\hline 1 & $a$ & 3371.5 & 359 & $03: 00$ & 06 & 1 & 0.088 \\
2 & $b$ & 3906.9 & 291 & $03: 40$ & 11 & 1 & 0.023 \\
3 & $c$ & 4193.5 & 309 & $02: 40$ & 05 & 5 & 0.007 \\
4 & $d$ & 4540.0 & 332 & $07: 00$ & 21 & 5 & 0.008 \\
5 & $e$ & 4925.0 & 374 & $06: 10$ & 16 & 8 & 0.006 \\
6 & $f$ & 5266.8 & 344 & $05: 30$ & 17 & 6 & 0.003 \\
7 & $g$ & 5789.9 & 289 & $05: 10$ & 16 & 6 & 0.005 \\
8 & $h$ & 6073.9 & 308 & $04: 00$ & 13 & 3 & 0.007 \\
9 & $i$ & 6655.9 & 491 & $05: 10$ & 20 & 8 & 0.009 \\
10 & $j$ & 7057.4 & 238 & $06: 20$ & 21 & 3 & 0.009 \\
11 & $k$ & 7546.3 & 192 & $03: 50$ & 12 & 5 & 0.002 \\
12 & $m$ & 8023.2 & 255 & $06: 00$ & 16 & 2 & 0.009 \\
13 & $n$ & 8484.3 & 167 & $04: 20$ & 09 & 3 & 0.006 \\
14 & $o$ & 9182.2 & 247 & $04: 32$ & 11 & 2 & 0.005 \\
15 & $p$ & 9738.5 & 275 & $01: 00$ & 10 & 2 & 0.004 \\
\hline
\end{tabular}

Notes.- Listed are the parameters of the BATC filters and statistics of our observation sample. Columns 1, 2 represent the ID of the BATC filters. Columns 3, 4 represent the central wavelengths and $F W H M$ of the 15 BATC filters, respectively. From Cols. 5 to 7 , we list the the total exposure time, number of images and number of calibration of each filter band. The last column gives the calibration error in magnitude.

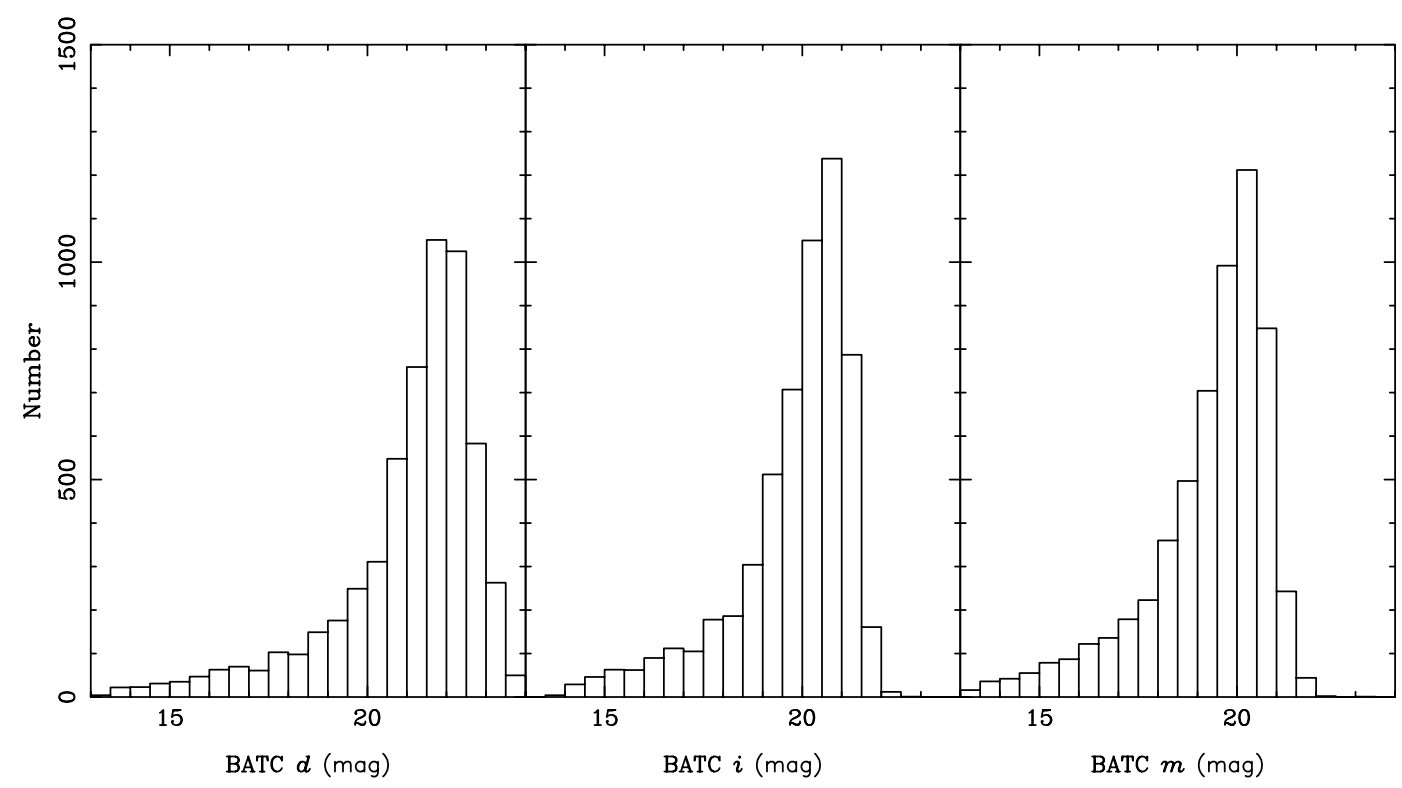

Fig. 1. The distribution of the BATC $d, i, m$ filter for 6000 objects in the BATC T329 field.

templates for the galaxies are the enlarged galaxy evolutionary library of Bruzual \& Charlot (1993), and the empirical template that is obtained through the averaged spectra of observed local field galaxies (Coleman 1980). Details about the classification of galaxies are given in Xia et al. (2002). The input library for stellar spectra is the Pickles (1998) catalog. This library consists of 131 flux-calibrated spectra, including all normal spectral types and luminosity classes at solar abundance, and metal-weak and metal-rich F-G dwarfs and $\mathrm{G}-\mathrm{K}$ giant components. Our sample may contain stars spread over a range of different metallicities. In Fig. 2, the two-color diagram $(d-i)$ versus $(i-m)$ is shown for our sample. Although the 15 filters are used in the object classification, the two-color diagram based only on the $d, i, m$ filters as an example shows that the scatter still exists in our sample. Most of stars lie in the mean main sequence track, and for those objects beyond the track, the scatter can be mainly due to the metallicity effect. Secondly, the analysis of deep star counts can be critically affected by faint galaxy contamination. At the magnitude limit of our data $(i \sim 21)$, galaxies outnumber stars. Although our multicolor photometric information is effective at discriminating stars from galaxies, some fainter objects still can be misclassified due to the increase in the observational error associated with the photometry of faint stars. The possibility of galaxy contamination is estimated to be less than $3 \%$. In addition, there is an effect called "non-simultaneous observation", and it will play an important role for long period variable stars (Chen 1996). However, this should not affect the final results derived 
by our sample, since the variable stars are relatively few. Here, we can only give the upper limit (less than 2\%) (Zhang 2002; private communication).

For those stars, the probability of belonging to a certain star class is computed by the SED fitting method. The standard $\chi^{2}$ minimization, i.e., computing and minimizing the deviations between photometric SED of a star and the template SEDs obtained with the same photometric system, is used in the fitting process. The minimum $\chi_{\min }^{2}$ indicates the best fit to the observed SED by the set of template spectra:

$\chi^{2}=\sum_{l=1}^{N_{\text {filt }}=15}\left[\frac{F_{\mathrm{obs}, l}-F_{\mathrm{temp}, l}-b}{\sigma_{l}}\right]^{2}$,

where $F_{\text {obs }, l}, F_{\text {temp }, l}$ and $\sigma_{l}$ are the observed fluxes, template fluxes and their uncertainty in filter $l$, respectively, and $N_{\text {filt }}$ is the total number of filters in the photometry, while $b$ is the mean magnitude difference between observed fluxes and template fluxes.

Thus, we can obtain the spectral types and luminosity classes for stars in the BATC survey. Due to the multicolor photometry, the classification can be relatively accurate. After knowing the stellar type, the photometric parallaxes can be derived by estimating absolute stellar magnitudes. We adopted the absolute magnitude versus stellar type relation for mainsequence stars from Lang (1992). The derived relation $M_{\mathrm{v}} \mathrm{vs}$. $(d-i)$ for the main sequence is shown in Fig. 3. In this figure, the scatter band in the relation $M_{\mathrm{v}}$ vs. $(d-i)$ is probably due to the larger uncertainty in the absolute magnitude determination only using two colors to express the information resulting from 15 colors.

A variety of errors affect the determination of stellar distances. The first source of errors could be from photometric uncertainty; the second from the misclassification that affects the derivation of absolute magnitude, but the misclassification should be small due to the multicolor photometry. For luminosity class $\mathrm{V}$, types $\mathrm{F}-\mathrm{G}-\mathrm{K}$, the absolute magnitude uncertainty is about $0.3 \mathrm{mag}$, and 0.8 or more for late $\mathrm{M}$ main sequence stars. In addition, there may be an error by the contamination of binary stars in our sample. We neglect the effect of binary contamination on distance derivation. For binaries with equal mass components, the distance will be assumed closer by a factor of $\sqrt{2}$. Due to the unknown but probable mass distribution in binary components, the effect is certainly less severe (Kroupa 1993; Ojha 1996). Since the BATC T329 field is at intermediate latitude $\left(b=49.80^{\circ}\right)$, we can neglect the influence of interstellar extinction in distance calculation.

\section{Space density distribution}

In previous work, various models have been developed to describe the stellar populations of the Galaxy. In general, these models were based on the assumption of a suitable spatial density distribution, and on the observational luminosity function and color-magnitude diagram for each stellar population (Bahcall \& Soneira 1984; Reid \& Majewski 1993) to fit the structural parameters by exploiting the measurements of colors and magnitudes. Here, on account of the use of the photometric parallaxes, we can make a direct evaluation of the spatial

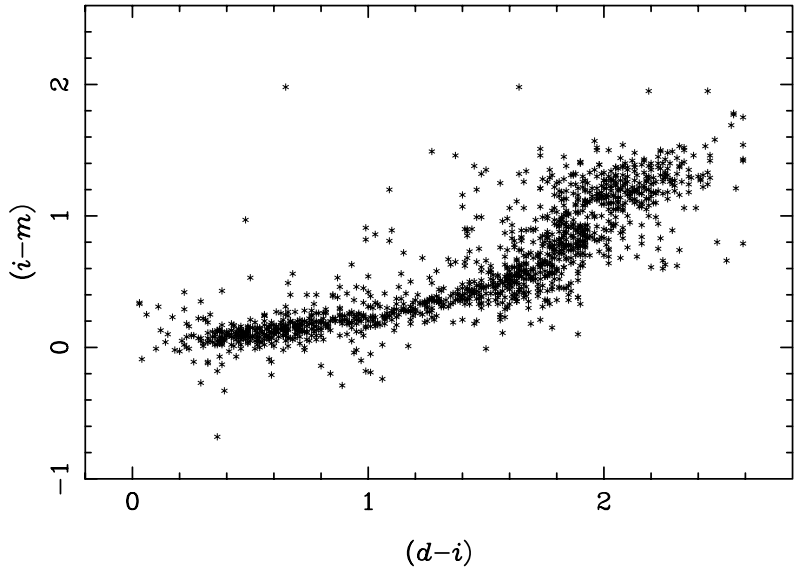

Fig. 2. The distribution of $(i-m)$ versus $(d-i)$ for the BATC T329 field down to the limiting magnitude, $i \leq 21$.

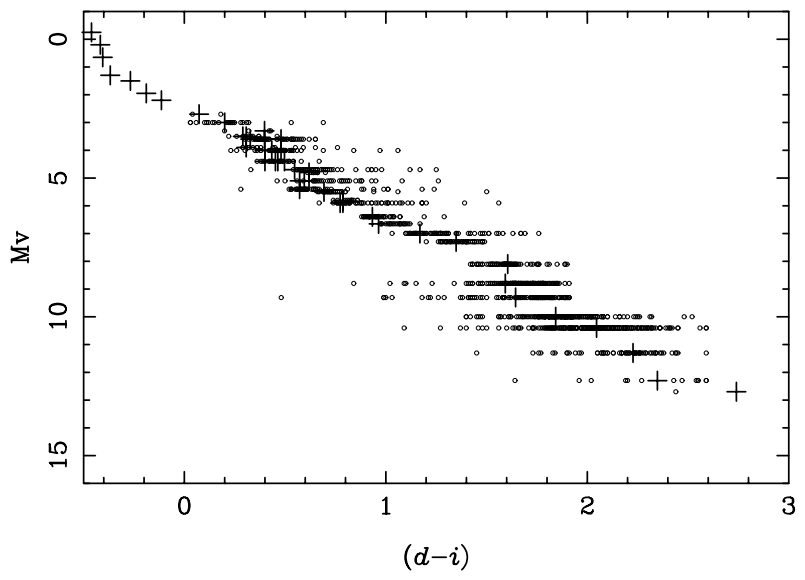

Fig. 3. $M_{\mathrm{v}}-(d-i) \mathrm{CMD}$ for the main sequence stars is shown. The open circles refer to stars of the BATC T329 field, and the crosses refer to stellar library of Pickles (1998).

density law. Rather than trying to fit the structure of the Galaxy in the observed parameter space of colors and magnitudes, we translate the observations into discrete density measurements at various points in the Galaxy.

As the starting point for our analysis, we consider models with three major components: a thin disk and a thick disk, both with exponential density profiles, and a halo component, with a deprojected de Vaucouleurs profile. Given a reliable stellar sample, the next step is to differentiate between member stars of the disk and those of the halo population. It is important to consider the volume-sampling effects inherent in any star count analysis. As described in Reid et al. (1993, 1996), the convolution of a monotonically-decreasing density law with the sampling volume leads to a "preferred" distance modulus dependent on the slope of the density law along the particular Galactic line of sight for a given stellar population. Since the density distribution of the disk population falls off faster with height above the plane than does that of the halo, and the former is sampled primarily at $m-M \sim 7 \mathrm{mag}$, while the latter lies preferentially at $m-M \sim 17$ mag. Thus, at a given apparent magnitude, the halo contributes more luminous (bluer) stars than the disk. In Fig. 4, we plot the color distribution of our 


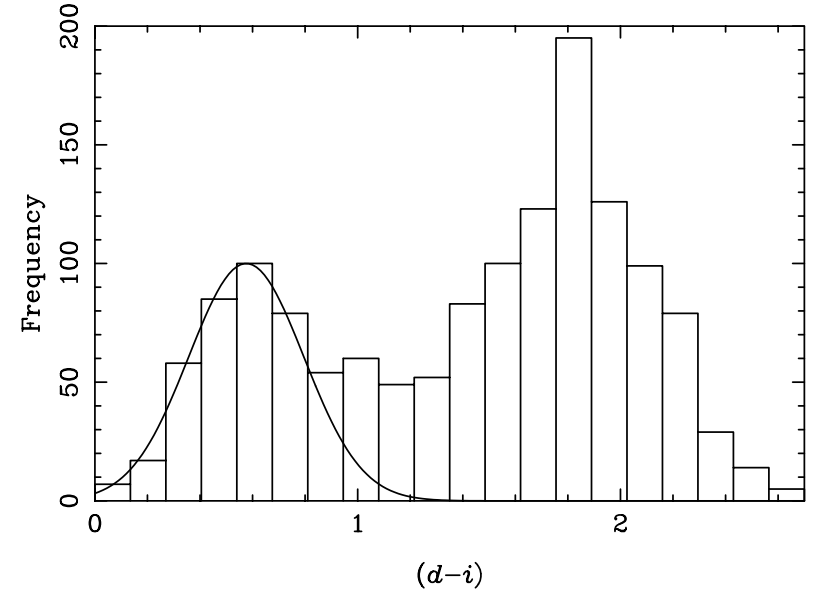

Fig. 4. Distribution of $(d-i)$ in the field, this bimodal distribution can be used to separate halo and disks by a color cut.

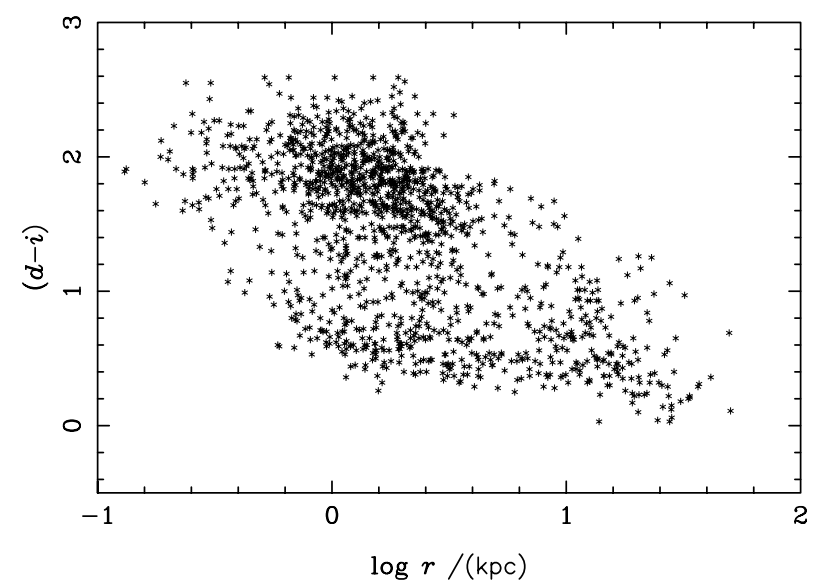

Fig. 5. Spatial distribution of $(d-i)$, and the photometric distances are derived according to the stellar type.

sample stars. From this figure, we can see the bimodal color distribution and the left peak is a close to a normal distribution. We use a Gaussian function to fit it and check the convergence by its left wing which is not contaminated by disk stars. We find that the bluer stars are dominated by halo stars which are supposed to be metal-poor, with a turnoff of $d-i \sim 0.9$. Beyond this point the contribution by disk stars becomes dominant. Therefore, a crude disk-halo separation can be drawn by color cut. We try to derive the structural parameters (e.g. scale height) of the thin and thick disk populations using our data set at intermediate latitude. For this we calculate the stellar space density as a function of distance from the Galactic plane. At first, we use the two dimensional distribution of stars in the $(d-i)$ vs. $\log r$ diagram (Fig. 5) to correct for this incompleteness. The nearest bins are assumed to be complete; for the incomplete bins we multiply iteratively by a factor given by the ratio of complete to incomplete number counts in the previous bin (Phleps et al. 2000). With the corrected number counts, the density in the $\log$ arithmetic space volume bins $V_{j}$ can then be calculated according to

$\rho_{j}=\frac{N_{j}^{\text {corr }}}{V_{j}}$

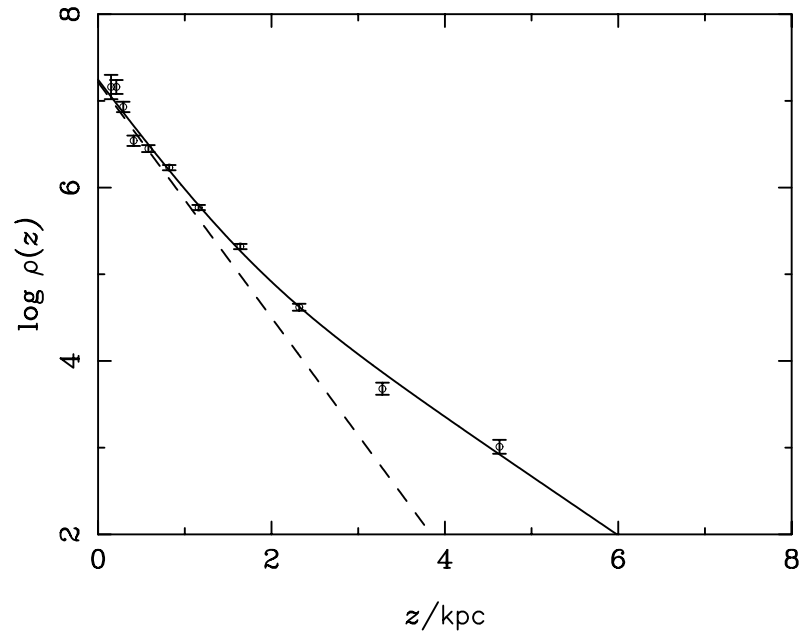

Fig. 6. Vertical density distribution of the disk stars $(d-i>0.9)$ in the field. The solid line is a fit with a superposition of two exponentials, the dashed line is the single exponential fit for the thin disk component.

here, $V_{j}=(\pi / 180)^{2}(\omega / 3)\left(r_{j+1}^{3}-r_{j}^{3}\right)$ is partial volume, $r_{j+1}$ and $r_{j}$ are the limiting distances; and $\omega$ is field size in square degrees.

\subsection{Density distribution in the disk}

We study the density distribution of the disk stars by taking stars of $(d-i) \geq 0.9$. The color cut is a crude separation between disk and halo, but we can gain a clear insight to the disk distribution due to the relatively less contamination by halo stars. Figure 6 shows the resulting density distribution of the disk stars in our field. The solid line represents a fit with a superposition of two exponentials, i.e., the function of the disk is:

$\rho(z)=n_{1} \exp \left(-z / h_{1}\right)+n_{2} \exp \left(-z / h_{2}\right)$,

$h_{1}$ and $h_{2}$ are the scale heights of the thin disk and thick disk, respectively, and the dashed line is the fit for the thin disk component. It is obvious that a single exponential disk is not a good fit for the disk component. Thus, the thick disk component in our Galactic model is indispensable to explain the observed star counts and color data. The comparison between data and simulations is made using a $\chi^{2}$-fit. The corresponding parameters can be given. The scale height of the thin disk $h_{1}$ is $320_{-15}^{+14} \mathrm{pc}$. The thick disk's scale height $h_{2}$ is $640_{-32}^{+30} \mathrm{pc}$, and the corresponding space number density normalization is $7.0 \pm 1 \%$ of the thin disk. The errors of scale heights and the corresponding space number density normalization are estimated at a $68 \%$ confidential level.

Some results for the thin disk scale height have been published in the literature. Most authors (Gilmore 1984; Bahcall \& Soneira 1984; Yoshii 1987; Reid \& Majewski 1993) derived a scale height of $325 \mathrm{pc}$ for old-disk stars; Chen (2001) used two large star count samples to derive the scale height of the thin disk to be 330 pc. However, Kuijken \& Gilmore (1989) derived a scale height of 249 pc for the old-disk stars. Haywood (1994) showed, from an analysis of numerous star counts towards the pole using his self-consistent evolutionary model, that the thin 


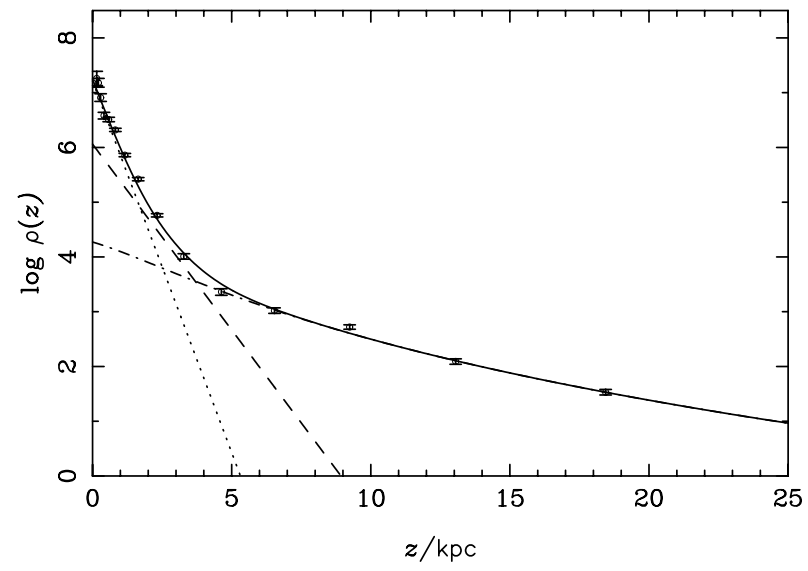

Fig. 7. Density distribution perpendicular to the Galactic plane, the dotted line shows the contribution of the thin disk component, the dashed line is the contribution of the thick disk, the dot-dashed line is a de Vaucouleurs law and the solid line the sum of the three.

disk scale height does not exceed 250 pc. Ojha (1999) also found that the scale height of the thin disk is $240 \mathrm{pc}$ based on an analysis of two star count samples. It is clear that our derived thin disk scale height $(320 \mathrm{pc})$ is consistent with the value presented by Chen (2001) and the classical value in the literature.

The thick disk density law can be reasonably modeled either by a exponential or by a density law close to $\operatorname{sech}^{2}(z)$, and star counts are unable to distinguish between these two hypothesis (Reylré \& Robin 2001). The thick disk vertical structure is generally described as exponential with scale heights varying between $480 \mathrm{pc}$ and $1500 \mathrm{pc}$ and local density between $1 \%$ to $15 \%$ relative to the thin disk. Because of the small proportion of the thick disk locally with regard to the thin disk, it is difficult to derive a accurate scale height and local density of the thick disk. In general, any values of $h_{z}$ in the range $480-1500 \mathrm{pc}$ and of local density in $1 \%-15 \%$ turn out to be acceptable.

The thick disk's scale height is anticorrelated with its local density when fitted simultaneously, and a small scale height is obtained in combination with high local density, while large scale height is associated with low local density (Robin et al. 1996; Chen 2001). Robin et al. (1996) derived a scale height of $h_{z}=760 \pm 50 \mathrm{pc}$ with a local density of $5.6 \pm 1.0 \%$ relative to the thin disk, and Ojha et al. (1999) presented a scale height of $790 \mathrm{pc}$ with a local density of $6.1 \%$ of the thin disk from a photometry and proper-motion survey in the two directions at intermediate latitude. Gilmore (1984) presented a scale height of $1300 \mathrm{pc}$ and local normalization of $2 \%$, Kuijken \& Gilmore (1989) derived a scale height of $1000 \mathrm{pc}$ and local normalization of $4 \%$. Chen (2001) gave a thick disk scale height between $580 \mathrm{pc}$ and $750 \mathrm{pc}$, with a local density of $13-6.5 \%$ of the thin disk. Our study gives a scale height of $640_{-32}^{+30} \mathrm{pc}$ and the corresponding space number density normalization is $7.0 \pm 1 \%$ of the thin disk. In Table 2, we summarize the main parameters relating to the vertical distribution of stars in these models. It is clear that our results favor the thick disk scale height presented by Chen et al. (2001), well below the original proposal of Gilmore et al. (1984).
Figure 7 gives the density distribution of all stars in the BATC T329 field. The dotted line shows the contribution of the thin disk component; the dashed line is the contribution of the thick disk; the dot-dashed line is a de Vaucouleurs law, and the solid line is the sum of the three components. It shows that the thick disk dominates star counts at distances between 1.5 and $4 \mathrm{kpc}$ over the galactic plane. Phleps (2000) derived that the thick disk provides the predominant contribution from $1.5 \mathrm{kpc}$ to $5.0 \mathrm{kpc}$. It showed that our thick disk result determination is in agreement with Phleps's (2000). However, photometric counts are not accurate enough to estimate the distances of stars at the turnoff with an accuracy of even a factor of two. It can also be seen that the corresponding plots fit the distribution of the halo stars up to distance of over $20 \mathrm{kpc}$ above the Galactic plane.

\subsection{The density law of the halo}

We use a de Vaucouleurs law for the halo component of the Galaxy. The de Vaucouleurs law is an empirical description of the density distribution of the galactic halo. The analytic approximation is:

$$
\begin{aligned}
\rho_{H}(z, b, l)= & \rho_{0} \frac{\exp \left[-10.093\left(\frac{R}{R_{\odot}}\right)^{1 / 4}+10.093\right]}{\left(\frac{R}{R_{\odot}}\right)^{(7 / 8)}} \\
& \times 1.25 \frac{\exp \left[-10.093\left(\frac{R}{R_{\odot}}\right)^{1 / 4}+10.093\right]}{\left(\frac{R}{R_{\odot}}\right)^{(6 / 8)}}, \\
& \times\left[1-0.08669 /\left(R / R_{\odot}\right)^{1 / 4}\right], \quad R \geq 0.03 R_{\odot}
\end{aligned}
$$

where $R=\left(x^{2}+z^{2} / \kappa^{2}\right)^{1 / 2}$ is Galactocentric distance, $\kappa$ is the axis ratio, $x=\left(R_{\odot}^{2}+d^{2} \cos ^{2} b-2 R_{\odot} d \cos b \cos l\right)^{1 / 2}, z=d \sin b$; $R_{\odot}=8 \mathrm{kpc}$ is the distance of the sun from the Galactic center, $b$ and $l$ are the Galactic latitude and longitude; the normalization factor $\rho_{0}$ is usually expressed as a percentage of the local spatial density of stars.

In our sample, disk stars greatly outnumber halo stars and it is therefore difficult to isolate halo stars, and we use the blue tail of the distribution (Fig. 4) $d-i<0.9$ mag for our star counts to distinguish the population of halo stars from the sample. Adopting a de Vaucouleurs $r^{1 / 4}$ law halo and a local density normalization $\rho_{0}=0.125 \%$, the $\chi^{2}$-curve for the axis ratio parameter as derived from the BATC T329 field is shown in Fig. 8. Our counts imply that the axial ratio of the stellar halo approximates 0.6 . This ratio agrees with the star count results of Larsen (1994) and is also consistent with a kinematic analysis, but it does not agree with the ratio from the Koo et al. (1986) surveys. The apparent discrepancy is probably due to the multi-component nature of the Galactic halo (Buser 1985). Some studies of the kinematics and abundance of both field stars and globular clusters show that the halo is better described as having two subpopulations-a flattened inner halo and a spherical outer halo (Siegel 2002). Additional support for dual-halo models can be drawn from the apparent dichotomy in 
Table 2. A comparison of the parameters obtained from different sources.

\begin{tabular}{c|c|cc|cccc}
\hline \hline Component & $\begin{array}{c}\text { Thin disk } \\
h_{1}(\mathrm{pc})\end{array}$ & $\begin{array}{c}\text { Thick disk } \\
h_{2}(\mathrm{pc})\end{array}$ & $\begin{array}{c}\text { Thick disk } \\
\text { Local normalization }\end{array}$ & $\begin{array}{c}\text { Halo } \\
\text { de Vaucouleurs } \\
\text { Local normalization }\end{array}$ & $\begin{array}{c}\text { axis ratio } \\
\text { power law } \\
\text { index }(n)\end{array}$ & axis ratio \\
\hline Bahcall (1984) & 325 & $\ldots$ & $\ldots$ & $0.15 \%$ & 0.8 & $\ldots$ & $\ldots$ \\
Gilmore (1984) & 325 & 1300 & $2 \%$ & $0.125 \%$ & 0.8 & $\ldots$ & $\ldots$ \\
Kuijken (1989) & 249 & 1000 & $4 \%$ & $\ldots$ & $\ldots$ & $\ldots$ & $\ldots$ \\
Ojha (1999) & 240 & 790 & $6.1 \%$ & $\ldots$ & $\ldots$ & $\ldots$ & $\ldots$ \\
Robin (2000) & $\ldots$ & 750 & $5.6 \%$ & $0.125 \%$ & $\ldots$ & 2.44 & 0.76 \\
Chen (2001) & 330 & $580-750$ & $13-6.5 \%$ & $\ldots$ & $\ldots$ & 2.5 & 0.55 \\
Siegel (2002) & 280 & $700-1000$ & $6-10 \%$ & $\ldots$ & 0.48 & $\ldots$ & $\ldots .6$ \\
Larsen (1994) & $\ldots$ & $\ldots$ & $7.0 \%$ & $0.125 \%$ & 0.58 & $\ldots$ & $\ldots$ \\
This work & 320 & 640 & & & $\ldots$ & $\ldots$ \\
\hline
\end{tabular}

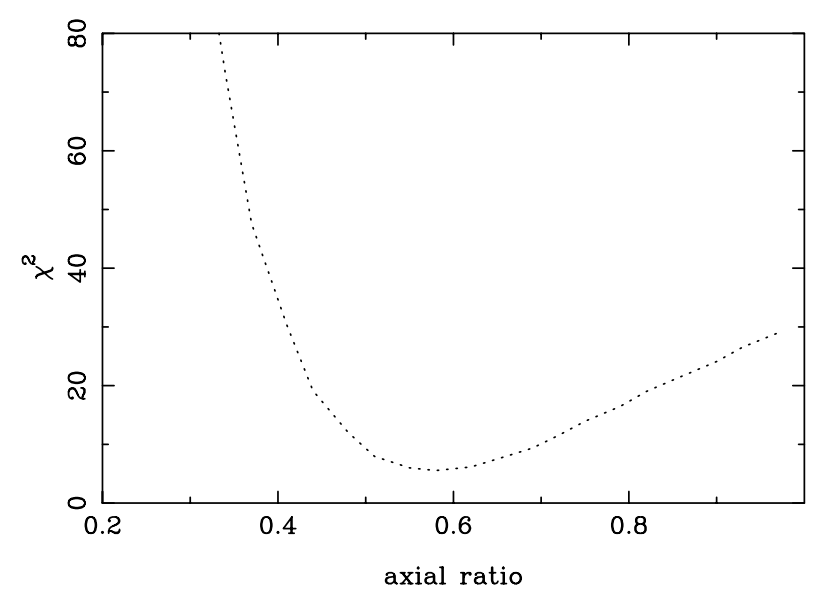

Fig. 8. $\chi^{2}$-curve for the axis ratio of de Vaucouleurs $r^{1 / 4}$ halo, derived from star count data of the BATC field.

detailed chemical abundance of halo stars (Nissen \& Schuster 1997). In a dual-halo model, nearby stars (Larsen 94; Chen 2001; Siegel 2002; this work) are dominated by the flattened inner halo while distant stars (Koo 1986; Bahcall 1984; Nissen 1997) are dominated by the round outer halo. Such models may resolve many of the disagreements in star count results.

Sources of uncertainty in our star counts include the effects of extinction and possible contamination by thick disk stars. Chen (2001) found that the redding for the BATC T329 field, $E_{B-V}<0.011$ and $A_{v} / E_{B-V}$ for the bluest band $a$ is 5.46, therefore, it yields $A_{v}$ (the BATC $a$ band) 0.06 and lower than our observation error 0.1 , so we can neglect its effect. A major uncertainty in the interpretation of our star counts is from a thick disk. However, the small sample prevents further conclusions about halo structure. We will recheck these results with forthcoming BATC data in other Galactic directions in the future.

\section{The stellar luminosity function}

The stellar luminosity function (SLF) gives the number of stars per cubic parsec of space per unit interval of absolute magnitude $M$ as a function of $M$. The SLF is a key input parameter for models of the stellar content of the Galaxy. The stellar samples are used to derive it, and counts of stars are estimated to be complete at distance (from the sun) limits of $24 \mathrm{kpc}$ and

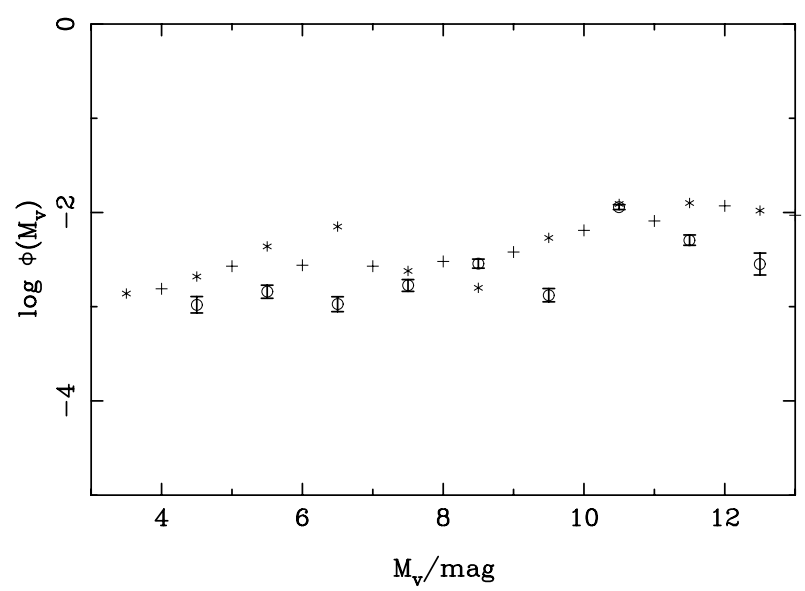

Fig. 9. The SLF of the thin disk stars. The circles represent the present determination, including error bars determined within the fit. The crosses show the local SLF of Jahreiß (1997a), and the stars are derived from Eggen (1983).

absolute magnitude limits of 12 mag. The stellar luminosity function differs in different parts of the Galaxy. Of the greatest interest is its determination in the thin disk. The stellar luminosity function for the thin disk stars can be calculated according to the knowledge of the density distribution function. We selected the nearby stars as thin-disk stars with distances less than $1.5 \mathrm{kpc}$. Beyond this point the contribution from thick disk and halo stars becomes dominant.

To investigate this possibility further, we make the following alternative estimate of the SLF. We first calculate the effective volume, $v_{\text {eff }}\left(M_{\mathrm{v}}\right)$, as a function of absolute magnitude by integrating the volume element as a function of the distance $r$ along the line of sight,

$v_{\text {eff }}\left(M_{\mathrm{v}}\right)=\omega \int_{R_{\min }}^{R_{\max }} v(r, b) r^{2} \mathrm{~d} r$,

here, the integration limits are

$R_{\min }=10^{0.2\left(14-M_{V}\right)-2.0}$

$R_{\max }=\min \left(1.5 \mathrm{kpc}, 10^{0.2\left(21-M_{V}\right)-2.0}\right)$,

and $v(r, b)$ is the distribution function of the thin disk, and it is normalized to unity at $z=0$. 
Table 3. The logarithmic luminosity function for disk stars in the neighborhood, where $v_{\text {eff }}$ is effective volume in $\mathrm{pc}^{3}, N$ is the number of stars in the absolute magnitude bin.

\begin{tabular}{cccc}
\hline \hline$M_{V}$ & $v_{\text {eff }}$ & $N$ & $\log \phi\left(M_{V}\right)$ \\
\hline$(4,5)$ & $2.383(4)$ & 25 & -2.98 \\
$(5,6)$ & $2.706(4)$ & 39 & -2.84 \\
$(6,7)$ & $2.820(4)$ & 30 & -2.97 \\
$(7,8)$ & $2.855(4)$ & 48 & -2.77 \\
$(8,9)$ & $2.864(4)$ & 82 & -2.54 \\
$(9,10)$ & $2.867(4)$ & 38 & -2.88 \\
$(10,11)$ & $2.387(4)$ & 272 & -1.94 \\
$(11,12)$ & $1.218(4)$ & 62 & -2.29 \\
$(12,13)$ & $4.935(3)$ & 14 & -2.55 \\
\hline
\end{tabular}

Finally, we divide the total number of stars in a given magnitude bin by the effective volume of that bin. The derived SLF is listed in Table 3 and in Fig. 9, in comparison with SLF derived by Eggen (1983) from a survey of proper motion selected stars with apparent magnitude $V<15$, and the SLF by Jahreiß $\&$ Wielen (1997) from the superior HIPPARCOS data for the local stellar population.

As can be seen from Fig. 9, the observed luminosity function in the BATC T329 field is in agreement with the local luminosity function as given by Eggen (1983) and by Jahreiß $\&$ Wielen (1997) only for three intervals, i.e.: $7<M_{\mathrm{v}}<8$, $8<M_{\mathrm{v}}<9$, and $10<M_{\mathrm{v}}<11$, whereas the observed SLF is lower than the Wielen (1997) standard for the other intervals. The low value of the present observed SLF at the brighter luminosity intervals is most probably due to the larger uncertainty in the absolute magnitude determination. It is noticeable that there is a dip at the faint luminosity intervals $11<M_{\mathrm{v}}<12$ and $12<M_{\mathrm{v}}<13$. It is possible that the dip is real at the faint magnitude end. In addition, the discrepancy at the faint magnitude intervals may come from the fact that the stars are too faint to be sampled in any significant number and uncertainties of the classification may introduce systematic errors that are hard to quantify.

\section{Conclusions}

Based on the BATC observation, we have analyzed the star counts with the help of a Galaxy model in order to parameterize the vertical distribution of stars in the Milky Way. The basic conclusions of this paper are:

1. Based on the BATC multicolor photometry and on the template SEDs, the stellar spectral types and luminosity classes can be obtained. Thus, the photometric parallaxes of the main sequence stars can be derived.

2. Using two exponential disks, we determine that the scale height of the thin disk is $320_{-15}^{+14} \mathrm{pc}$ and the thick disk scale height is $640_{-32}^{+30} \mathrm{pc}$ with a corresponding space number density normalization $7.0 \pm 1 \%$ of the thin disk.

3. Adopting a de Vaucouleurs $r^{1 / 4}$ law halo and a local density normalization $\rho_{0}=0.125 \%$, the observed counts yield a axial ratio of $c / a \leq 0.6$, implying a more flattened halo. Our study suggests that it is possible that the halo has two subpopulations-a flattened inner halo and a spherical outer halo in the Milky Way.

4. Based on this knowledge of the density distribution, we determined the stellar luminosity function (SLF) for the thin disk stars, with distances less than $1.5 \mathrm{kpc}$. Beyond this point the contribution by thick disk and halo stars become dominant.

In general, these preliminary test results are in remarkable agreement with the majority of independent recent determinations. Since our data comprise a large field, with a multicolor filter system including 15 intermediate-band filters, this finding confirms in a satisfactory way similar results found from other projections. Of course, the structural parameter values derived from the BATC survey data critically depend on the reliability of the basic model. While this model needs to be refined in many respects in the future, it can provide a suitable framework for interpreting the BATC data in its present form.

Acknowledgements. We would like to thank the anonymous referee for his/her insightful comments and suggestions that improved this paper. The BATC Survey is supported by the Chinese Academy of Sciences, the Chinese National Natural Science Foundation under the contract No. 10273012, the Chinese State Committee of Sciences and Technology. This work has been supported by the National Key Basic Research Science Foundation (NKBRSF TG199075402). We also thank the assistants who helped with the observations for their hard work and kind cooperation.

\section{References}

Bahcall, J. N., \& Soneira, R. M. 1980, ApJS, 44, 73

Bahcall, J. N., \& Soneira, R. M. 1984, ApJS, 55, 67

Bahcall, J. N., Ratnatunga, K. U., Buser, R., et al. 1985, ApJ, 299, 616

Bruzual, A. G., \& Charlot, S. 1993, ApJ, 405, 538

Buser, R., \& Kaeser, U. 1985, A\&A, 145, 1

Coleman, G. D., Wu, C. C., \& Weedman, D. W. 1980, ApJS, 43, 393

Chen, B. 1997, ApJ, 491, 181

Chen, B., Figueras, F., Torra, J., et al. 1999, A\&A, 352, 459

Chen, B., Stoughton, C., \& Smith, A. 2001, ApJ, 553, 184

Chen, A. B. 1996, Ph.D. Thesis, Institute of Astronomy, National Central University, Chung-Li, Taiwan

Chen, A. B., Méndez, R. A., Tsay, W.-S., \& Lu, P. K. 2001, AJ, 121, 309

Eggen, O. J. 1983, ApJS, 51, 183

Fan, X. H., Burstein, D., Chen, J.-S., et al. 1996, AJ, 112, 628

Fukugita, M., Ichikawa, T., Gunn, J. E., et al. 1996, AJ, 111, 1748

Gilmore, G. 1984, MNRAS, 207, 223

Haywood, M. 1994, A\&A, 282, 444H

Jahreiß, H., \& Wielen, R. 1997a, HIPPARCOS'97. Presentation of the HIPPARCOS and TYCHO catalogues and first astrophysical results of Hipparcos space astrometry mission., ed. B. Battrick, M. A. C. Perryman, \& P. L. Bernacca, ESA SP-402, Noordwyk, 675

Jenkner, H., Lasker, B. M., Sturch, C. R., et al. 1990, AJ, 99, 2082

Kuijken, K., \& Gilmore, G. 1989, MNRAS, 239, 605

Koo, D. C., \& Kron, R. G. 1982, A\&A, 105, 107

Koo, D. C., Kron, R. G., \& Cudworth, K. M. 1986, PASP, 98, 285

Kron, R. G. 1980, ApJS, 43, 305

Kroupa, P., Tout, C. A., \& Gilmore, G. 1993, MNRAS, 262, 545

Lang, K. R. 1992, Astrophysical Data I, Planets and stars (Berlin: Spinger-Verlag)

Larsen, J. A., \& Humphrey, R. M. 1994, ApJ, 436, L149

Méndez, R. A., \& Minniti, D. 1996, MNRAS, 283, 666 
Norris, J. E. 1998, in Galaxy Evolution: Connecting the Distant Universe with the local Fossil Record, ed. M. Spite (Dordrecht: Kluwer Academic Publishers), in press

Nissen, P. E., \& Schuster, W. J. 1997, A\&A, 326, 751

Ojha, D. K., Bienayme, O., Mohan, V., \& Robin, A. C. 1999, A\&A, 351,945

Ojha, D. K., Bienaymé, O., Robin, A. C., \& Crézé, M. 1996, A\&A, 311,456

Oke, J. B., \& Gunn, J. E. 1983, ApJ, 266, 713

Phleps, S., Meisenheimer, K., Fuchs, B., \& Wolf, C. 2000, A\&A, 356, 108

Pickles, A. J. 1998, PASP, 110, 863P

Reid, I. N., \& Majewski, S. R. 1993, ApJ, 409, 635

Reid, I. N., Yan, L., Majewski, S., et al. 1996, AJ, 112, 1472

Reylé, C., \& Robin, A. C. 2001, A\&A, 373, 886
Robin, A. C., Haywood, M., \& Crézé, M. 1996, A\&A, 305, 125

Robin, A. C., Reylé, C., \& Crézé, M. 2000, A\&A, 359, 103

Stetson, P. B. 1987, PASP, 99, 191S

Siegel, M. H., Majewski, S. R., \& Reid, I. N. 2002, ApJ, 578, 151

Wielen, R., Jahrei $\beta$, H., \& Kruger, R. 1983, in The Nearby Stars and the Stellar Luminosity Function, ed. A. G. Davis Philip, \& Upgren, Davis Press, Schenectady, N.Y., IAUC, 76, 163

Wyse, R. F. G., \& Gilmore, G. 1989, MNRAS, 239, 605

Xia, L. F., Zhou, X., Ma, J., et al. 2002, PASP, 114, 1349

Yan, H. J., \& Burstein, D., 2000, PASP, 112, 691

Yoshii, Y., \& Ishida, K. 1987, AJ, 93, 323Y

Zhang, X. B., Deng, L., Tian, B., \& Zhou, X. 2002, AJ, 123, 1548

Zhou, X., Jiang, Z. J., Xue, S. J., Wu, H., Ma, J., \& Chen, J. S. 2001, ChJAA, 1, 372

Zhou, X., Jiang, Z., Ma, J., et al. 2003, A\&A, 397, 361 